\title{
THE FOSSIL CONTINENTAL MOLLUSKS IN THE UPPER FRESHWATER MOLASSE (MIDDLE MIOCENE) OF THE DISTRICTS OF BIBERACH, RAVENSBURG AND NEU-ULM, GERMANY
}

\author{
RODRIGO B. SALVADOR \\ Staatliches Museum für Naturkunde Stuttgart, Rosenstein 1, 70191, Stuttgart, Germany. \\ salvador.rodrigo.b@gmail.com \\ VOLKER J. SACH \\ In der Talwiese 2, 72488, Sigmaringen, Germany. \\ BÁRBARA L. VALENTAS-ROMERA \\ Instituto de Biociências da Universidade de São Paulo, Rua do Matão, travessa 14, 321, \\ 05508-090, São Paulo, Brazil.
}

\begin{abstract}
The present work is a taxonomical study of the continental mollusks collected in several fossil outcrops in the districts of Biberach and Ravensburg (SE Baden-Württemberg state) and Neu-Ulm (SW Bavaria state), Germany, by one of the authors (V.J.S.) in the years 1983-2013. All these fossil sites are of Middle Miocene age (European Mammal Neogene zones MN 5 and MN 6) and belong to the Upper Freshwater Molasse (“Obere Süßwassermolasse” or OSM), in the North Alpine Foreland Basin of southern Germany. The molluscan fauna of these sites counts with 17 gastropod and three bivalve species: Tinnyea laureae, Pomatias sp., Bithynia sp., Theodoxus sp., Galba cf. G. dupuyiana, Lymnaea dilatata, Gyraulus applanatus, Planorbarius cornu, Opeas minutum, Clausiliidae indet., Triptychia sp., Archaeozonites costatus, Klikia sp., Pseudochloritis incrassata, Palaeotachea renevieri, Megalotachea silvana, Deroceras sp., Margaritifera flabellata, Unio kirchbergensis and Sphaerium aff. S. rivicola. Finally, a few paleoenvironmental remarks are offered.
\end{abstract}

Key words: Bivalvia, European Mammal Neogene Zones MN5 and MN 6, Gastropoda, OSM, Pulmonata.

RESUMO - O presente trabalho é um estudo taxonômico dos moluscos continentais coletados em diversos afloramentos fossilíferos nos distritos de Biberach e Ravensburg (SE do Estado de Baden-Württemberg) e Neu-Ulm (SO do Estado de Bayern), Alemanha, por um dos autores (V.J.S.) durante os anos 1983-2013. Tais afloramentos datam do Mioceno Médio (zonas neógenas de mamíferos europeus MN5 e MN 6), pertencendo ao grupo “Obere Süßwassermolasse” (OSM) da bacia Alpina do Norte. A malacofauna dessas localidades conta com 17 espécies de gastrópodes e três de bivalves: Tinnyea laureae, Pomatias sp., Bithynia sp., Theodoxus sp., Galba cf. G. dupuyiana, Lymnaea dilatata, Gyraulus applanatus, Planorbarius cornu, Opeas minutum, Clausiliidae indet., Triptychia sp., Archaeozonites costatus, Klikia sp., Pseudochloritis incrassata, Palaeotachea renevieri, Megalotachea silvana, Deroceras sp., Margaritifera flabellata, Unio kirchbergensis e Sphaerium aff. S. rivicola. Adicionalmente, são oferecidos alguns comentários sobre o paleoambiente das localidades estudadas.

Palavras-chave: Bivalvia, zonas neógenas de mamíferos europeus MN 5 e MN 6, Gastropoda, OSM, Pulmonata.

\section{INTRODUCTION}

Sach (1999) described several Middle Miocene fossil sites in the district of Biberach, Baden-Württemberg state, SW Germany, with his main focus being the lithology, sedimentology, biostratigraphy and the fossil mammals of the region. This author also listed many mollusk-bearing sites, but, since they were not the objective of his study, he did not provide a formal treatment of them. These mollusks are also listed, but not described nor figured, in a recently published catalogue of Early/Middle Miocene fossil sites in southern Germany (Sach, 2014). This catalogue also brings species lists of a few other newly discovered mollusk-bearing fossil sites in the neighboring districts of Ravensburg, in Baden-Württemberg, and Neu-Ulm, in Bavaria State (Figure 1), preliminarily listed by Sach (2014). Herein is presented a taxonomical analysis of these fossil continental mollusks, which are represented by 17 gastropod and three bivalve species, alongside with some paleoenvironmental remarks. 


\section{MATERIAL AND METHODS}

The mollusk-bearing fossil sites in the districts of Biberach, Ravensburg and Neu-Ulm can be seen in Figure 1, as named by Sach $(1999,2014)$. The Middle Miocene fossil sites studied here are detailed below.

\section{Biberach District}

Wannenwaldtobel 1: 4801'50.88'”N, 0950'01.14”'E; boulder horizon of the Ries impact ("Brockhorizont" in the original), $603.5 \mathrm{~m}$ above sea level (a.s.l.); Mammal Neogene zones MN 5/6 (transition).

Wannenwaldtobel 2: coordinates as Wannenwaldtobel 1; calcareous marl and marlstone horizon ("Kalkmergel" in the original), $610.0 \mathrm{~m}$ a.s.l.; additional fine-sand horizon with bivalves (Margaritifera flabellata) at $~ 609$ m a.s.l.; Mammal Neogene zones MN 5/6 (transition).

Tobel Oelhalde-Nord: 4802'24.95”N, 0949'52.03’'E; two fine-grained sand horizons with reworked OSM components, 604.6 m and 612.5 m a.s.l.; Mammal Neogene zones MN 5/6 (transition).

Tobel Oelhalde-Süd: $48^{\circ} 02^{\prime} 16.08^{\prime \prime} \mathrm{N}, 09^{\circ} 49^{\prime} 47.06^{\prime \prime} \mathrm{E}$; finesand horizon with reworked OSM components, $596.3 \mathrm{~m}$ a.s.l.; additional fine-grained sand horizon with bivalves (Margaritifera flabellata) at $600.2 \mathrm{~m}$ a.s.l.; Mammal Neogene zones MN 5/6 (transition).

Edelbeuren-Schlachtberg: 4805’21.38’’N, 1001'17.71’'E; two fine-grained sand horizons with reworked OSM components, 583 m a.s.l.; Mammal Neogene zone MN 5.

Binnrot: 4803'49.01'N, $10^{\circ} 03^{\prime}$ 07.94”'E; fine-sand horizons with reworked OSM components, 571.3-572.7 a.s.l.; additional sandy horizon with bivalves (Sphaerium aff. rivicola) at $\sim 577 \mathrm{~m}$ a.s.l.; Mammal Neogene zone MN 5.

Bonlanden: 48 $04{ }^{\prime} 03.71^{\prime \prime} \mathrm{N}, 10^{\circ} 04^{\prime} 11.04$ ' $\mathrm{E}$; fine-sand horizon with reworked OSM components, 564.9 m a.s.l.; Mammal Neogene zone MN 6.

Edelbeuren-Maurerkopf: 4805'40.82’’N, 1001'51.93’'E; two fine-sand horizons with reworked OSM components, 559.5-563.0 m a.s.l.; Mammal Neogene zone MN 5.

Heselsberg (Ochsenhausen-Heselsberg): 4804'11.19”'N, 9॰57’23.48”'E; fine-gravel horizon ("Erolzheimer Sande” at "Baustelle Remmele" in the original) with reworked OSM components, $\sim 607 \mathrm{~m}$ a.s.l.; and $48^{\circ} 04^{\prime} 05.33^{\prime \prime} \mathrm{N}$, 9०57'27.72”'E; fine-sand horizon ("Baustelle Harsch” in the original) with reworked OSM components, $\sim 620 \mathrm{~m}$ a.s.l.; Mammal Neogene zone MN 5 (both fossil sites).

Auttagershofen: 48 $11^{\circ} 56.49^{\prime \prime} \mathrm{N}, 1^{\circ} 01^{\prime} 04.74$ '” E; calcareous marl horizon, $531 \mathrm{~m}$ a.s.l.; Mammal Neogene zone MN 5.

\section{Ravensburg District}

Burgerbachtobel 1: 4748'03.26”, N 9²6'59.22'”E (close to the border of Ravensburg and Bodenseekreis districts); calcareous marl and fine-sand horizons with reworked OSM components, 580 m a.s.l.; Mammal Neogene zone MN 6; Schmalegger Tobel: 47²4'25.21'”, N 9'32'16.14”'E; fine-sand horizons with reworked OSM components, $\sim 510 \mathrm{~m}$ above sea level; Mammal Neogene zone MN 6.
Lattentobel: 4749'49.70’, N 9²5'57.63'”E; fine-sand horizons with reworked OSM components, $\sim 610 \mathrm{~m}$; Mammal Neogene zone MN 6.

\section{Neu-Ulm District}

Altenstadt-Untereichen: 4809'29.47'”N, 1006'39.93'”; finesand horizons with reworked OSM components, 562-566 m a.s.l.; additional fine-sand horizon with bivalves (Margaritifera flabellata) at 570 m a.s.l.; Mammal Neogene zone MN 5.

The largest portion of the fossil material studied here derives from the localities Wannenwaldtobel 2, EdelbeurenMaurerkopf, Burgerbachtobel 1 and Altenstadt-Untereichen. Together, these four sites have so far yielded numerous fossils of mammals, other vertebrate groups, non-mollusk invertebrates (insects, crustaceans and ostracods) and plants (Sach, 1999, 2014). There are some additional sites in these districts that also bear mollusk fossils (Sach, 1999, 2014), but the material was too fragmentary and was not collected and thus, could not be studied here. As such, the records for the following sites could not be confirmed in the present work: Biberach: Awengen (Lymnaea sp., Cepaea sp.) and Oberstetten (Cepaea sp.); Ravensburg: Ibacher Tobel, Horber-/Staigertobel, Aichertobel and Weiler Sandkeller (Unio sp. vel Margaritifera sp.) and Höll-Tobel (?Tropidomphalus sp., Margaritifera cf. flabellata). The sites Schwendi-Tobel and Brunnentobel, in Ravensburg, despite harboring mollusks (Sach, 1999, 2014), were excluded from the present work, since their location and stratigraphical data remain unknown.

All the material from the above mentioned fossil sites, which is housed in the collection of the Staatliches Museum für Naturkunde Stuttgart (SMNS; Stuttgart, Germany), was analyzed for the present work. A list of the examined material follows each species description. The specimens were collected during the years of 1983 to 2013 by V.J. Sach; for more details, see Sach (1999). Systematic arrangements follow Bouchet et al. (2005) and Nordsieck (2014). Selected specimens were examined by scanning electronic microscopy (SEM) in the SMNS; shell measurements were obtained either with a digital caliper or with the aid of computer software. The following shell measurements abbreviations are used throughout the text: $\mathbf{H}$, shell height; $\mathbf{D}$, shell greatest width; $\mathbf{h}$, operculum height; L, shell length (bivalves).

\section{GEOLOGICAL SETTING}

All the studied fossil sites date from the Middle Miocene (European Mammal Neogene zones MN 5 and MN 6) and belong to the stratigraphical unit known as the Upper Freshwater Molasse (“Obere Süßwassermolasse” in German, or OSM). More specifically: four sites (Bonlanden, Burgerbachtobel 1, Lattentobel and Schmalegger Tobel) are deemed to belong to the MN 6 zone; three sites (Wannenwaldtobel 1+2, Tobel Oelhalde-Süd and Tobel Oelhalde-Nord) belong to the transitional period MN 5/6; the remaining sites date from the MN 5 zone. For a more thorough 


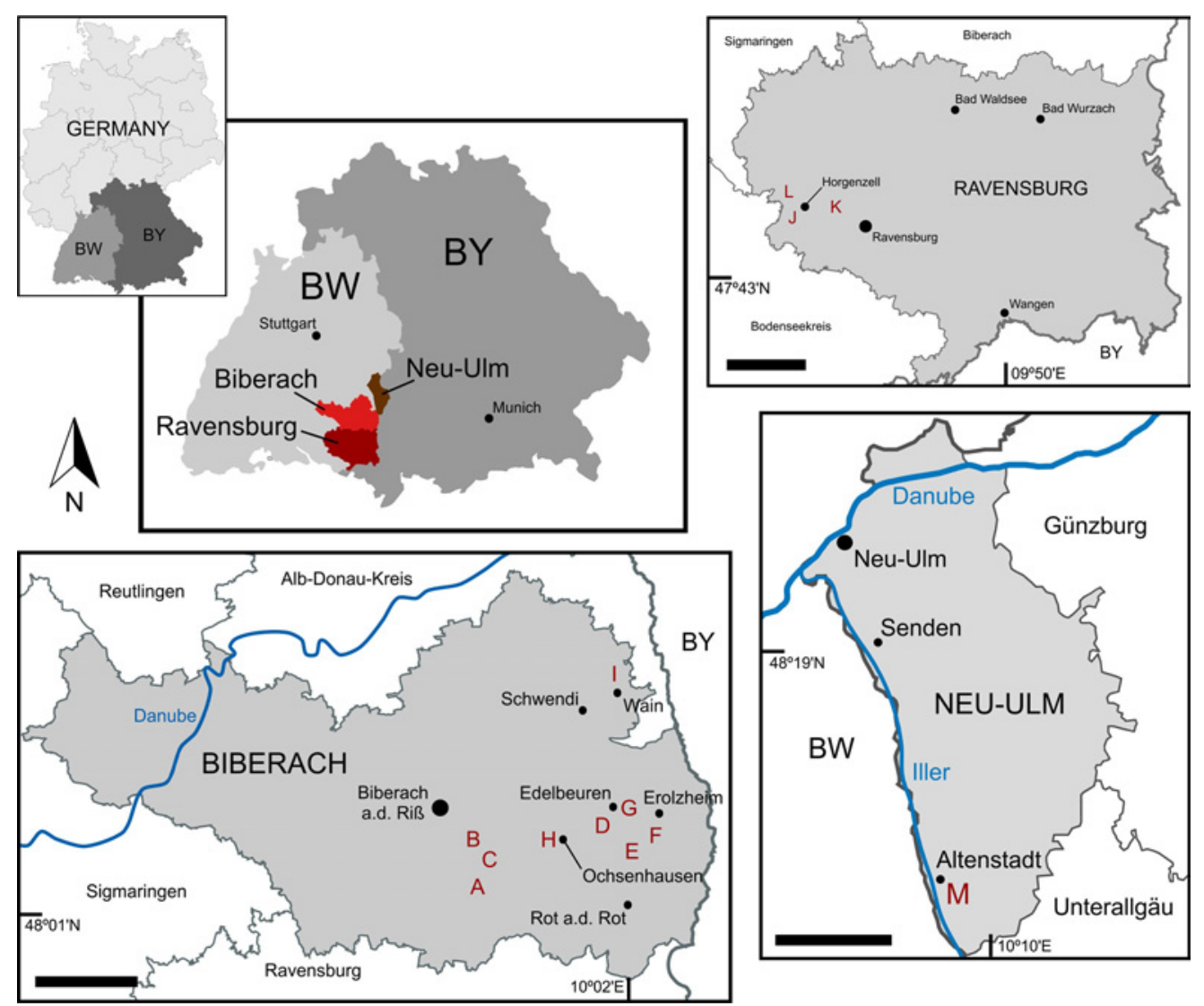

Figure 1. Map of the districts of Biberach and Ravensburg, Baden-Württemberg state (BW), and Neu-Ulm, Bavaria state (BY), Germany, indicating the Middle Miocene fossil sites (names according to Sach 1999, 2014). A, Wannenwaldtobel 1+2; B, Tobel Oelhalde-Nord; C, Tobel OelhaldeSüd; D, Edelbeuren-Schlachtberg; E, Binnrot; F, Bonlanden; G, Edelbeuren-Maurerkopf; H, Heselsberg; I, Auttagershofen; J, Burgerbachtobel 1; K, Schmalegger Tobel; L, Lattentobel; M, Altenstadt-Untereichen. Scale bars $=10 \mathrm{~km}$.

description of each fossil site, as well as the lithology of the fossiliferous sediments, their lithostratigraphic position and biostratigraphy, see Sach $(1999,2014)$ and Sach et al. (2003).

\section{SYSTEMATIC PALEONTOLOGY}

Class GASTROPODA Cuvier, 1795

Clade CAENOGASTROPODA Cox, 1960

Superfamily CERITHIOIDEA Fleming, 1822

Family PACHYCHILIDAE P. Fischer \& Crosse, 1892

Tinnyea Hantken, 1887

Tinnyea lauraea (Mathéron, 1843)

(Figures 2A-B)

1843 Melania lauraea Mathéron, p. 291, pl. 36, figs. 23-24. 1927 Melanatria escheri turrita: Berz \& Jooss, p. 206.

1953 Brotia (Tinnyea) escheri: Papp, p. 128, pl. 3, fig. 29; Schlickum, 1976, p. 4, pl. 1, fig. 9; Mikuz \& Pavsic, 2000, p. 44, pl. 1, figs. 1-8.

2014 Brotia escheri: Sach, p. 23.

Material examined. SMNS 101648 (seven specimens).

Occurrence. Altenstadt-Untereichen.
Description. Shell medium-sized to large, turreted, with regularly growing whorls. Protoconch not preserved. Teleoconch sculpture by parallel oblique axial ribs on first whorls (distance between axial ribs roughly twice their width) and by both axial and spiral cords on the remaining whorls (distance between cords roughly equal to three times their width). First spiral cord on each whorl stronger, with spikelike protuberances when it meets with axial ribs. Whorls profile convex. Suture well-marked, but not too deep.

Discussion. As pointed out by Kadolsky (1995), the name $T$. escheri (Brongniart, 1823) is a nomen nudum. The first valid name for the species is T. laureae. This species is known in the fossil record of Central Europe from the Oligocene to the Pliocene, but is rarer in the early and middle Miocene (Harzhauser et al., 2002). Nevertheless, it is known from the coeval nearby locality of Oggenhausen (Berz \& Jooss, 1927). Paleoecological remarks. This species is known from freshwater and oligohaline sediments (Kókay. 2006).

Superfamily LITTORINOIDEA Children, 1834 Family POMATIIDAE Newton, 1891

Pomatias Studer, 1789

Pomatias sp. 
Material examined. SMNS 101678 (one operculum).

Occurrence. Burgerbachtobel 1.

Discussion. A single fragmentary operculum can be assigned to Pomatias by its spiral growth pattern, but identification beyond genus level is presently not possible.

Superfamily RISSOOIDEA Gray, 1847

Family BITHYNIIDAE Gray, 1857

Bithynia Leach, 1818

Bithynia sp.

(Figure 2C)

1999 Bithynia sp.: Sach, p. 24; Sach et al., 2003, p. 6; Sach, 2014, p. 27.

Material examined. SMNS 101539 (>100 operculum). Occurrence. Edelbeuren-Maurerkopf.

Discussion. Only opercula remain; their overall shape and concentric growth pattern indicate the genus Bithynia, but identification at species level is impossible.

Paleoecological remarks. Recent Bithynia live in richly vegetated slow moving or standing waters, some are even found in temporary water bodies (Welter-Schultes, 2012).

Clade NERITIMORPHA Golikov \& Starobogatov 1975 Family NERITIDAE Lamarck, 1809

Theodoxus Montfort, 1810

Theodoxus sp.

(Figure 2D)

2014 Theodoxus crenulatus: Sach, p. 25.

Material examined. SMNS 101649 (one specimen).

Occurrence. Altenstadt-Untereichen.

Discussion. The color pattern is preserved in this single fragment; this distinctive pattern allows the assignment to the genus Theodoxus. Despite a more precise identification at species level not being possible, it should be remarked that Theodoxus crenulatus (Klein, 1853) is a very common and widespread species in the middle and late Miocene of Central Europe and exhibits a great variation in color pattern (e.g. Harzhauser et al., 2012).

Paleoecological remarks. The habitat range of recent Theodoxus species is ample, so its use for paleoecological inferences is very limited; nevertheless, all species are intolerable to drought and seem to prefer rocky substrates (Bandel, 2001; Zettler et al., 2004; Bunje, 2005; WelterSchultes, 2012).

PULMONATA Cuvier, 1814

Clade HYGROPHILA

Superfamily LYMNAEOIDEA Rafinesque, 1815

Family LYMNAEIDAE Rafinesque, 1815
Galba Schrank, 1803

Galba cf. G. dupuyiana (Noulet, 1854)

(Figure 2E)

1999 Galba sp.: Sach, p. 16; Sach et al., 2003, p. 9; Sach, 2014, p. 37.

1999 Lymnaea cf. L. armaniacensis: Sach, p. 24; Sach et al., 2003, p. 6; Sach, 2014, p. 27.

Material examined. SMNS 101540 (43 specimens), 101541 (one specimen), 101542 (one specimen), 101543 (one specimen), 101544 (eight specimens), 101545 (nine specimens).

Occurrence. Wannenwaldtobel 2, Edelbeuren-Maurerkopf and Auttagershofen.

Description. Shell small $(\mathrm{H}<1 \mathrm{~cm})$, lymnaeiform; shell width $\sim 1 / 2$ shell height. Transition proto- to teleoconch unclear. Teleoconch smooth, except for well-marked growth lines. Suture deep, well-marked. Whorls profile convex. Aperture oval, narrow, elongated. Peristome simple. Imperforate.

Discussion. Only broken and/or deformed spires remain in the present material, but they are reminiscent of Galba dupuyiana, a common species known throughout the middle Miocene of West and Central Europe (Kókay, 2006). The present specimens compare especially well with the taller and more elongated forms reported from the German early/middle Miocene sites of Oggenhausen and Sandelzhausen (Böttcher et al., 2009; Salvador \& Rasser, 2014).

Paleoecological remarks. The single recent European species, Galba truncatula (O.F. Müller, 1774), inhabits both permanent and temporary water bodies, usually on the margins (Chapuis et al., 2007; Welter-Schultes, 2012).

Lymnaea Lamarck, 1799

Lymnaea dilatata (Noulet, 1854)

(Figures 2F-G)

1854 Limnea dilatata Noulet, p. 107.

1921 Limnaea (Radix) dilatata: Ehrat \& Jooss, p. 4.

1923 Radix (Radix) socialis dilatata: Wenz, p. 1277; Seemann, 1926, p. 92.

1999 Lymnaea dilatata: Sach, p. 16; Fischer, 2000, p. 136, figs. 1-2; Binder, 2002, p. 165, pl. 1, fig. 7a; Sach et al., 2003, p. 6; Binder, 2004, p. 192, pl. 1, fig. 7; Kowalke \& Reichenbacher, 2005, p. 630, figs. 9.4-9.5); Sach, 2014, p. 23; Salvador \& Rasser, 2014, p. 189, figs. 8-9).

1999 Lymnaea sp.: Sach, p. 20.

1999 Tudorella conica: Sach, p. 24; Sach et al., 2003, p. 6. 2006 Radix dilatata: Kókay, p. 52, pl. 17, fig. 14; Neubauer et al., 2014, supplementary material 1.

Material examined. SMNS 101598 ( $~ 80$ specimens), 101599 ( 50 specimens), 101600 (one specimens), 101601 (three specimens), 101602 (one specimen), 101603 (two specimens), 101604 (six specimens), 101605 (three specimens), 101606 
(one specimen), 101647 (seven specimens), 101680 (one specimen), 101682 (one specimen).

Occurrence. Wannenwaldtobel 2, Tobel Oelhalde-Süd, Bonlanden, Heselsberg, Edelbeuren-Maurerkopf, Burgerbachtobel 1 and Altenstadt-Untereichen.

Description. Shell large, lymnaeiform; spire acuminated, proportionately small. Transition proto- to teleoconch unclear. Teleoconch smooth, except for growth lines. Suture well-marked. Whorls profile slightly convex. Body whorl proportionately large. Peristome simple. Imperforate.

Discussion. Lymnaea dilatata is known from the whole Miocene of West and Central Europe (Binder, 2004; Kókay, 2006). Some authors (e.g. Wenz, 1923; Kókay, 2006) have placed the species in the genus Radix Montfort, 1810, but, as remarked by Fischer (2000), it is more reminiscent of Lymnaea. The single specimen previously identified as Pomatias conicus (Klein, 1853) by Sach (1999) and Sach et al. (2003) is a spire apex of L. dilatata.

Paleoecological remarks. Recent Lymnaea thrive in richly vegetated, shallow standing or slow-flowing waters, being commonly found in temporary water bodies (Glöer, 2002; Welter-Schultes, 2012).

Superfamily PLANORBOIDEA Rafinesque, 1815 Family PLANORBIDAE Rafinesque, 1815

Gyraulus Charpentier, 1837

Gyraulus applanatus (Thomae, 1845)

(Figures 2H-I)

1845 Planorbis applanatus Thomae, p. 150.

1851 Planorbis dealbatus Braun, p. 1134.

1923 Gyraulus (Gyraulus) trochiformis applanatus: Wenz, p. 1579.

1923 Gyraulus (Gyraulus) trochiformis dealbatus: Wenz, p. 1591.

1970a Gyraulus trochiformis applanatus: Schlickum, p. 148, pl. 10, fig. 6; Schlickum, 1970b, p. 180.

1964 Gyraulus trochiformis dealbatus: Schlickum, p. 15, pl. 2, fig. 35; Steininger et al., 1973, p. 451, pl. 9, figs. 11a,b; Reichenbacher, 1989, pl. 1, fig. 11; Kókay, 2006, p. 57, pl. 19, fig. 15.

2005 Gyraulus applanatus: Kowalke \& Reichenbacher, p. 631, figs. 9.1-9.3; Kókay, 2006, p. 56, pl. 19, figs. 13-14; Neubauer et al., 2014, supplementary material 1.

1995 Gyraulus dealbatus: Kadolsky, p. 40, fig. 47; Binder, 2004, p. 193, pl. 2, figs. 1a-c; Neubauer et al., 2014, supplementary material 1; Salvador \& Rasser, 2014, p. 192, figs. 16-23.

Material examined. SMNS 101544 (17 specimens), 101545 ( 30 specimens), 101546 ( 40 specimens), 101547 (one specimen).

Occurrence. Wannenwaldtobel 2 and Edelbeuren-Maurerkopf.
Description. Shell very small (D $<5 \mathrm{~mm}$ ), pseudodextral, planispiral; shell height $\sim 1 / 3$ shell width. Protoconch sculpture not preserved; transition to teleoconch unclear. Teleoconch smooth, except for growth lines. Spire depressed. Suture deep, well-marked. Whorls somewhat rapidly growing, especially body whorl. Body whorl rounded, with very faint keel on median portion. Aperture arrowhead-shaped. Peristome simple, sharp. Umbilicus wide, shallow.

Discussion. The present specimens compare well with Gyraulus applanatus by the aperture shape, rapidly growing whorls and a rounded body whorl profile with a faint keel on its median portion. This species has not been reported by Sach (1999, 2014) for any of the localities. Gyraulus applanatus is known from the very early to middle Miocene of Germany and Austria (Gottschick \& Wenz, 1916; Kadolsky, 1995; Binder, 2004; Kowalke \& Reichenbacher, 2005). There is some morphological variation within this species and transitional forms between it and G. dealbatus, as reported by previous authors (Gottschick \& Wenz, 1916; Kowalke \& Reichenbacher, 2005; Salvador \& Rasser, 2014). This led Kowalke \& Reichenbacher (2005) to synonymize both species. The present specimens from Biberach are mostly juvenile or fragmentary; the small number of adult specimens does not seem to present much variation.

Paleoecological remarks. Recent Gyraulus are found in many habitats, limiting their use in paleoecological analysis. Still, they usually inhabit shallow richly-vegetated standing or slow-flowing waters, sometimes even temporary water bodies (Welter-Schultes, 2012).

Planorbarius Duméril, 1806

$$
\text { Planorbarius cornu (Brongniart, 1810) }
$$

(Figure 2J)

1810 Planorbis cornu Brongniart, p. 371, pl. 22, fig. 6.

1923 Coretus cornu cornu: Wenz, p. 1426.

1966 Planorbarius cornu: Schlickum, p. 326, pl. 13, fig. 27; Schlickum, 1970a, p. 149, pl. 10, fig. 7; Reichenbacher, 1989, p. 172, pl. 1, fig. 10; Binder, 2004, p. 193, pl. 2, figs. 2-3; Neubauer et al., 2014, supplementary material 1; Salvador \& Rasser, 2014, p. 193, figs. 26-28.

1999 Coretus cf. C. cornu: Sach, p. 16.

1999 Coretus sp. Sach, p. 17.

2006 Planorbarius cornu cornu: Kókay, p. 58, pl. 20, fig. 6; Böttcher et al., 2009, p. 239, figs. 2.4-2.6.

2003 Planorbarius cf. P. cornu: Sach et al., p. 6; Sach, 2014, p. 23.

Material examined. SMNS 101571 (>100 specimens), 101572 (>100 specimens), 101573 (three specimens), 101574 (>100 specimens), 101575 (13 specimens), 101576 (one specimen), 101577 (two specimens), 101578 (one specimen), 101579 (one specimen), 101580 (four specimens), 101581 (five specimens), 101646 (eight specimens).

Occurrence. Wannenwaldtobel 2, Tobel Oelhalde-Nord, Tobel Oelhalde-Süd, Edelbeuren-Schlachtberg, Bonlanden, Edelbeuren-Maurerkopf and Altenstadt-Untereichen. 
Description. Shell large, sinistral, planispiral; shell height $~ 1 / 3$ shell width. Spire very depressed. Suture deep, well-marked. Whorls regularly growing. Whorl profile rounded. Aperture rounded. Umbilicus very wide, deep. Unfortunately, due to poor preservation, proto- and teleoconch sculpture cannot be observed. Discussion. The present specimens, despite the poor preservation, compare well with Planorbarius cornu, a morphologically variable species known from the middle Eocene to the late Miocene of France, Germany (especially abundant in the OSM) and Czech Republic (Schlickum, 1970a; Kókay, 2006). As pointed out by Harzhauser et al. (2014), this long time span could indicate the presence of more than one species. Until this species complex has been the target of revisionary work, we prefer a more conservative identification as $P$. cornu.

Paleoecological remarks. Recent Planorbarius are found in typically richly-vegetated standing or slow-moving waters (Glöer, 2002; Welter-Schultes, 2012).

Clade STYLOMMATOPHORA A. Schmidt, 1855 Superfamily ACHATINOIDEA Swainson, 1840

Family SUBULINIDAE P. Fischer \& Crosse, 1877

Opeas Albers, 1850

Opeas minutum (Klein, 1853)

(Figure 2K)

1853 Bulimus minutus: Klein, p. 212, pl. 5, fig. 9.

1923 Opeas minutum: Wenz, p. 872; Seemann, 1926, p. 92; Kókay, 2006, p. 80, pl. 30, fig. 16; Harzhauser et al., 2014, p. 28, pl. 9, figs. 3-5, 7.

1976 Opeas (Opeas) minutum: Schlickum, p. 14, pl. 3, fig. 46.

Material examined. SMNS 101551 (one specimen).

Occurrence. Edelbeuren-Maurerkopf.

Description. Shell small, with regularly growing whorls. Protoconch large, rounded, dome-shaped, apparently smooth; transition to teleoconch unclear. Teleoconch apparently smooth. Whorls profile slightly convex. Suture well-marked but not too deep.

Discussion. Opeas minutum is easily diagnosed by the small size of its subulinid shell. Although this single specimen is a spire apex, it compares extremely well with material from other Miocene fossil sites in Germany. The species is known from the early/middle Miocene of Hungary, Germany, Austria and Switzerland (Schlickum, 1976; Kókay, 2006; Harzhauser et al., 2014) and has not been reported by Sach $(1999,2014)$ for the present locality.

Paleoecological remarks. Recent Opeas are found in tropical and subtropical regions worldwide (Zilch, 1959-1960; Willig, 2013).

Superfamily CLAUSILIOIDEA J.E. Gray, 1855

Family CLAUSILIIDAE Gray, 1855

Clausiliidae indet.

(Figures 2L-M)

2003 Clausiliidae indet.: Sach et al., p. 6; Sach, 2014, p. 27.
Material examined. SMNS 101549 (one specimen), 101550 (four specimens).

Occurrence. Edelbeuren-Maurerkopf.

Discussion. The sinistral shell and rounded whorl profile (Figure 2L), as well as the sculpture pattern on the apertural fragment (Figure 2M), point to a clausiliid. Unfortunately, with the present material a more precise determination is not possible.

Paleoecological remarks. Nordsieck (2007) suggests that clausiliids up to the middle Miocene were mostly wooddwelling animals, preferring humid and warm environments.

\section{Family FILHOLIIDAE Wenz, 1923}

Triptychia Sandberger, 1875

Triptychia sp.

(Figure 2N)

Material examined. SMNS 101548 (one specimen).

Occurrence. Edelbeuren-Maurerkopf.

Description. Shell sinistral, with conical profile. Protoconch dome-shaped; transition to teleoconch unclear. Teleoconch sculpture not preserved. Whorl profile slightly convex. Suture deep, well-marked, slightly incised.

Discussion. The conical sinistral shell with its flat whorl profile agrees very well with the genus Triptychia. Nevertheless, it is presently impossible to proceed further than this in the classification of the material.

Paleoecological remarks. It is usually suggested that filholiids were mostly wood-dwelling animals, preferring humid and warm forests (Schnabel, 2007, and references therein).

\section{Superfamily ZONITOIDEA Mörch, 1864 Family ZONITIDAE Mörch, 1864}

Archaeozonites Sandberger, 1872

Archaeozonites costatus Sandberger, 1875

(Figures 2O-P)

1875 Archaeozonites costatus: Sandberger, p. 604; Schlickum, 1976, p. 18, pl. 5, fig. 66; Rasser et al., 2013, p. 440.

1921 Zonites (Aegopis) costatus: Ehrat \& Jooss, p. 3; Wenz, 1923, p. 254; Seemann, 1926, p. 91.

1999 Valvata sp.: Sach, p. 22; Sach, 2014, p. 31.

2006 Aegopis costatus: Kókay, p. 77, pl. 29, figs. 11-12.

2002 Miozonites costatus: Binder, p. 168, pl. 1, figs. 14-16, pl. 3, fig. 8, pl. 7, fig. 3, pl. 8, fig. 4; Harzhauser et al., 2014, p. 33, pl. 10, figs. 13-19.

Material examined. SMNS 101556 (three specimens), 101557 (one specimen), 101558 (one specimen), 101559 (three specimen).

Occurrence. Edelbeuren-Schlachtberg, Binnrot and Edelbeuren-Maurerkopf. 
Description. Shell large (D $>2 \mathrm{~cm}$ ), helicoid, with broad conical spire and regularly growing whorls. Protoconch $(\sim 1$ whorl) flattened, sculptured by fine riblets that grow coarser towards teleoconch. Teleoconch sculptured by numerous strong parallel prosocline ribs; distance between ribs equal to rib's width; ribs apparently absent on lower portion of whorls. Suture weakly marked. Whorls profile flattened; body whorl with a strong keel. Aperture oval. Umbilicus broad, deep.

Discussion. Despite the somewhat distorted preservation of the body whorl and aperture, the present specimens compare fittingly with Archaeozonites costatus, a species known from the early and middle Miocene of Central Europe (Kókay, 2006) and common in the Silvana-beds ("Silvanaschichten") of southwestern Germany (MN 5-6; Schlickum, 1976). The single specimen of Valvata sp. reported by Sach $(1999,2014)$ is a misidentification of a fragment of $A$. costatus.

Paleoecological remarks. As a fossil genus, any paleoecological inference is tentative; still, Lueger (1981) considered that Archaeozonites laticostatus (Sandberger, 1885), also from the Miocene of Central Europe, lived under leaves or between rocks in moist forests.

Superfamily HELICOIDEA Rafinesque, 1815 Family ELONIDAE Gittenberger, 1977

Klikia Pilsbry, 1895

Klikia sp.

(Figure 2Q)

1999 Klikia coarctata: Sach, p. 24; Sach et al., 2003, p. 6; Sach, 2014, p. 27.

Material examined. SMNS 101552 ( 85 specimens), 101645 (four specimens), 101681 (one specimen).

Occurrence. Edelbeuren-Maurerkopf, Heselsberg and Altenstadt-Untereichen.

Description. Shell medium-sized $(\mathrm{D}>1 \mathrm{~cm})$, depressed, multispiral. Suture well-marked, moderately deep. Whorls closely-packed, regularly increasing in size. Aperture crescent-shaped. Peristome reflexed.

Discussion. Despite the extremely poor preservation, the shell's discoid shape, size and the large number of narrowly coiled whorls indicate the genus Klikia. Nevertheless, identification beyond genus level is impossible.

Paleoecological remarks. Lueger (1981) suggested that species in this fossil genus preferred drier environments.

Family HELICIDAE Rafinesque, 1815

Pseudochloritis Boettger, 1909

Pseudochloritis incrassata (Klein, 1853)

(Figures 3A-C)

1846 Helix inflexa Klein [non von Zieten, 1832], p. 71, pl. 1, fig. 12.
1853 Helix incrassata Klein, p. 208, pl. 5, fig. 6.

1923 Tropidomphalus (Pseudochloritis) incrassatus incrassatus: Wenz, p. 510; Seemann, 1926, p. 91; Schlickum, 1976, p. 16, pl. 4, fig. 56.

1980 Tropidomphalus (Pseudochloritis) incrassatus incrassatus: Gall, p. 59.

1999 Tropidomphalus sp.: Sach, p. 16; Sach et al., 2003, p. 9. 1999 Tropidomphalus aff. zellii: Sach, p. 21; Sach et al., 2003, p. 6; Sach, 2014, p. 23.

2006 Tropidomphalus (Pseudochloritis) incrassatus: Kókay, p. 90, pl. 34, figs. 12-14.

2008 Pseudochloritis incrassata: Binder, p. 172, pl. 3, figs. 2-4, pl. 6, fig. 2; Rasser et al., 2013, p. 434, pl. 4, fig. 12; Harzhauser et al., 2014, p. 35, pl. 12, figs. 9-16, 19-24; Höltke \& Rasser, 2015, p. 1, figs. 4.3, 5.1-5.2, 6.11-6.12.

2008 Tropidomphalus (Pseudochloritis) incrassatus: Górka, p. 107, figs. 3.1-3.4.

Material examined. SMNS 101582 (28 specimens), 101583 (10 specimens), 101584 (one specimen), 101585 (four specimens), 101586 (five specimens), 101587 (three specimens), 101588 (one specimen), 101589 (one specimen), 101590 (one specimen), 101591 (two specimens), 101592 (one specimen), 101593 (two specimens), 101594 (two specimens), 101595 (two specimens), 101596 (two specimens), 101597 (>100 specimens), 101607 (one specimen), 101624 (three specimens), 101644 (eight specimens).

Occurrence. Wannenwaldtobel 1, Wannenwaldtobel 2, Tobel Oelhalde-Nord, Tobel Oelhalde-Süd, EdelbeurenSchlachtberg, Binnrot, Edelbeuren-Maurerkopf, Heselsberg, Schmalegger Tobel and Altenstadt-Untereichen.

Description. Shell large (D $>2 \mathrm{~cm}$ ), $\sim 4 \frac{1}{2}$ whorls, helicoid to disc-shaped, with flattened spire. Protoconch (11/4 whorl) flattened, large in relation to following whorl; sculptured by fine striae dotted with weak papillae; transition to teleoconch unclear. Teleoconch sculptured by thickened growth lines and irregular weak furrows, with regularly arranged papillae. Suture deep, well-marked. Body whorl enlarged, slightly bent downwards, with conspicuous constriction right before the aperture ("extralabial depression" sensu Binder 2008). Aperture prosocline; no callus apparent. Peristome markedly thickened and reflexed, slightly covering umbilicus. Umbilicus narrow to wide.

Discussion. The present specimens conform well to P. incrassata, a species known from the middle Miocene of Central Europe (Binder, 2008; Salvador, 2014). There is some uncertainty regarding the position of this genus, which is either assigned to Helicidae (Ariantinae) or to Elonidae (Zilch, 1959-1960; Binder, 2008; Moser, et al., 2009; Nordsieck, 2014; Höltke \& Rasser, 2015).

Paleoecological remarks. Based on the bent down body whorl and prosocline aperture, Binder (2008) considered the shell an adaptation for ground-dwelling (by reducing water loss in drier environments). Similarly, Moser et al. (2009) suggested that the genus inhabited drier and more open habitats, having a "way of life like strong-shelled Balkanese representatives of Ariantinae”. 
Palaeotachea Jooss, 1912

Palaeotachea renevieri (Maillard, 1892)

(Figures 2R-T)

1892 Helix (Macularia) renevieri Maillard, p. 43, pl. 3, fig. 18. 1923 Cepaea renevieri: Wenz, p. 652.

1954 Cepaea cf. C. renevieri: Zöbelein, p. 156.

1999 Cepaea sp.: Sach, p. 23; Sach, 2014, p. 31.

Material examined. SMNS 101560 (34 specimens), 101561 (24 specimens), 101562 (three specimens), 101563 (one specimen), 101564 (two specimens), 101565 (two specimens), 101566 (three specimens), 101567 (one specimen), 101568 (one specimen), 101569 (five specimens), 101570 (four specimens).

Occurrence. Tobel Oelhalde-Nord, Tobel OelhaldeSüd, Bonlanden, Edelbeuren-Maurerkopf, Heselsberg, Auttagershofen and Burgerbachtobel 1.

Description. Shell medium-sized (D > $1 \mathrm{~cm}$ ), 41/4 whorls, helicoid, with overall flattened profile and very faint keel; whorls quickly but regularly growing; shell length $~ 2 / 3$ its width. Protoconch flattened; transition to teleoconch unclear. Suture well-marked. Body whorl slightly bent downwards near the aperture. Aperture small, prosocline, circular. Peristome lightly thickened and slightly reflexed. Imperforate. On one specimen (SMNS 101563) it is possible to observe, under UV light, vestiges of three colored fine parallel spiral bands, the topmost one on the middle to upper portion of the whorl, the other two regularly spaced on the basal portion of whorl.

Discussion. Palaeotachea renevieri can be identified by its small size, a small circular aperture, a thickened but not reflexed peristome and a somewhat flattened shell profile with a faint keel. The species is known from the middle and late Miocene of Southern Germany and Switzerland (Wenz, 1923; Zöbelein, 1954). The observed trifasciate banding pattern is recurrent in Miocene helicids (e.g. Górka, 2008; Salvador, 2013).

Paleoecological remarks. As a fossil genus, paleoecological implications are not possible. Recent Cepaea Held, 1838 and allied species inhabit a broad range of habitats (WelterSchultes, 2012).

Megalotachea Pfeffer, 1930

Megalotachea silvana (Klein, 1853)

(Figures 2U-W)

1853 Helix silvana Klein, p. 205, pl. 5, fig. 2.

1923 Cepaea silvana silvana: Wenz, p. 667; Schlickum, 1976, p. 17, pl. 4, figs. 62-63; Reichenbacher, 1989, p. 165, pl. 2, figs. 17-19.

1999 Cepaea silvana: Sach, p. 16; Sach et al., 2003, p. 6; Kókay, 2006, p. 93, pl. 36, figs. 2-3; Rasser et al., 2013, p. 440; Sach, 2014, p. 23.
Material examined. SMNS 101608 (>100 specimens), 101609 (one specimen), 101610 (two specimens), 101611 (one specimen), 101612 (one specimen), 101613 (two specimens), 101614 (one specimen), 101615 (two specimens), 101616 (two specimens), 101617 (three specimens), 101618 (four specimens), 101619 (six specimens), 101620 (five specimens), 101621 (three specimens), 101622 (eight specimens), 101623 (34 specimens), 101625 (19 specimens), 101626 (35 specimens), 101627 (10 specimens), 101643 (16 specimens).

Occurrence. Wannenwaldtobel 1, Wannenwaldtobel 2, Tobel Oelhalde-Nord, Tobel Oelhalde-Süd, EdelbeurenSchlachtberg, Binnrot, Bonlanden, Edelbeuren-Maurerkopf, Heselsberg, Auttagershofen, Burgerbachtobel 1 and Altenstadt-Untereichen.

Description. Shell large (D > 2 cm), 41/4-41/2 whorls, helicoid; whorls regularly growing. Protoconch flattened, smooth; transition to teleoconch unclear. Teleoconch smooth, except for growth lines. Whorl profile flat on first whorls; more convex on later ones. Suture well-marked. Body whorl slightly bent downwards. Aperture prosocline, crescent-shaped; callus faint. Peristome markedly thickened and reflexed. Imperforate.

Discussion. The present specimens compare fittingly with Cepaea silvana, a common species in the German Early/ Middle Miocene, mainly due to their more rounded aperture and their greatly reflexed peristome. The specimens show a broad size range: $\mathrm{H}=14.9 \pm 2.0 \mathrm{~mm}(\max =19.0 \mathrm{~mm}$, $\min =13.0 \mathrm{~mm}), \mathrm{D}=21.9 \pm 1.9 \mathrm{~mm}(\max =28.0 \mathrm{~mm}$, $\min$ $=19.2 \mathrm{~mm}$ ). Still, the poor preservation of the fossils, with many internal molds and deformed specimens, precludes a precise description of morphological variation in other shell characters.

Paleoecological remarks. Same as Palaeotachea renevieri, above.

Superfamily LIMACOIDEA Lamarck, 1801

Family AGRIOLIMACIDAE H. Wagner, 1935

Deroceras Rafinesque, 1820

Deroceras sp.

(Figure 3D)

1999 Limax sp.: Sach, p. 16; Sach et al., 2003, p. 6; Sach, 2014, p. 27.

Material examined. SMNS 101553 (eight specimens), 101554 (>100 specimens), 101555 (22 specimens), 101677 ( 40 specimens).

Occurrence. Wannenwaldtobel 2, Edelbeuren-Maurerkopf and Burgerbachtobel 1.

Description. Shell small $(\mathrm{H}<1 \mathrm{~cm})$, vestigial, oval; shell width $~ 2 / 3$ length. Nucleus slightly bent laterally; nucleus region higher than remainder of shell. Shell with marked concentric growth lines.

Discussion. The vestigial shells of slugs are of very limited 


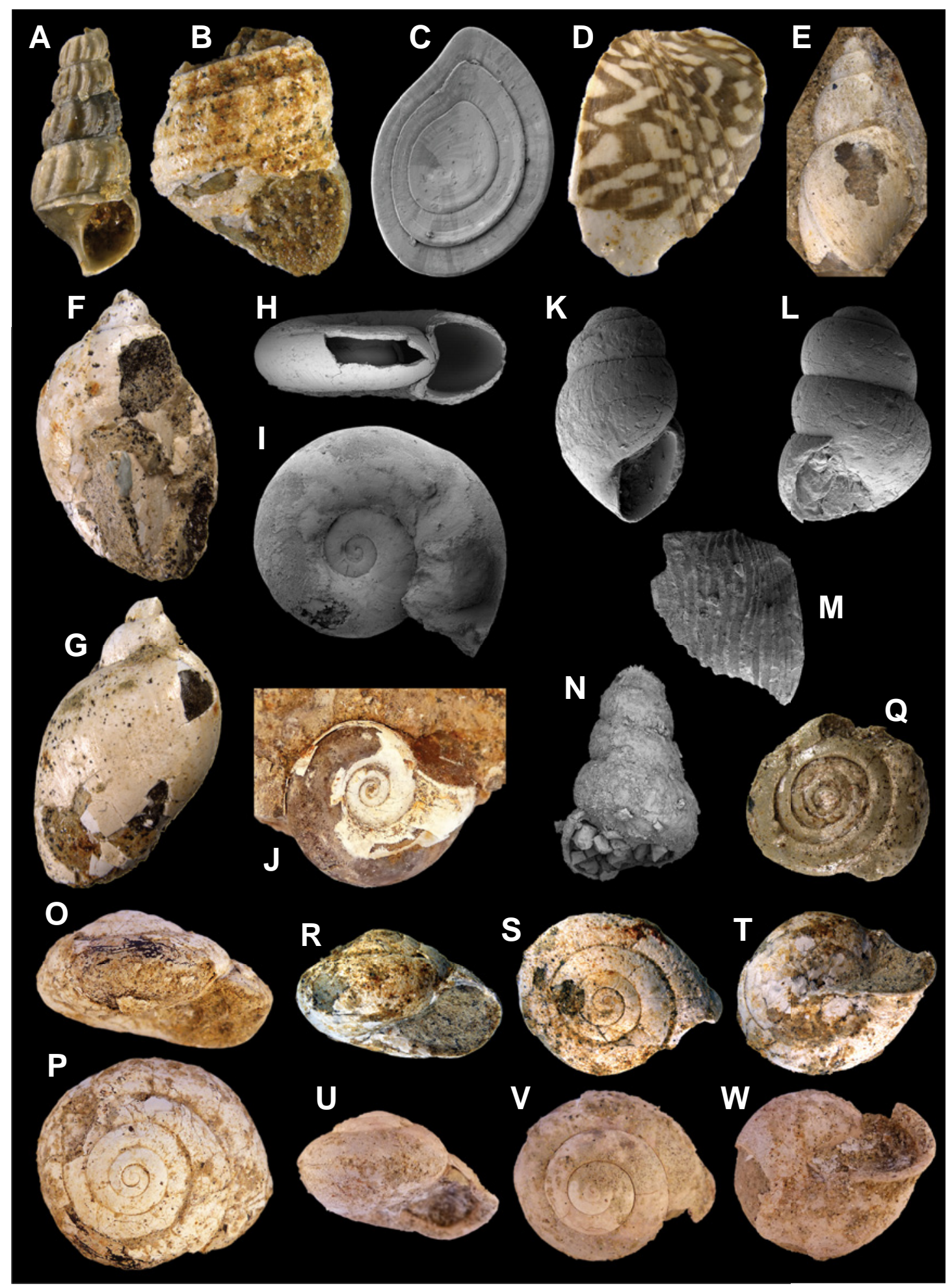

Figure 2. A, Tinnyea lauraea (SMNS 101648, specimen \#1, from Altenstadt-Untereichen; H=11.3 mm). B, Tinnyea lauraea (SMNS 101648, specimen \#2, from Altenstadt-Untereichen; $\mathrm{H}=16.0 \mathrm{~mm}$ ). C, Bithynia sp., operculum (SMNS 101539, from Edelbeuren-Maurerkopf; $\mathrm{h}=3.7$ $\mathrm{mm}$ ). D, Theodoxus sp. (SMNS 101649, from Altenstadt-Untereichen; fragment width $=4.5 \mathrm{~mm}$ ). E, Galba cf. G. dupuyiana (SMNS 101540, from Wannenwaldtobel 2; H = $8.2 \mathrm{~mm}$ ). F-G, Lymnaea dilatata (SMNS 101601, from Wannenwaldtobel 2; H = $17.8 \mathrm{~mm}, \mathrm{D}=11.1 \mathrm{~mm}$ ). H, Gyraulus applanatus (SMNS 101546, specimen \#1, from Wannenwaldtobel 2; D = $2.5 \mathrm{~mm}$ ). I, G. applanatus (SMNS 101546, specimen \#2, from Wannenwaldtobel 2; $\mathrm{D}=2.9 \mathrm{~mm}$ ). J, Planorbarius cornu (SMNS 101573, from Wannenwaldtobel 2; $\mathrm{D}=19.0 \mathrm{~mm}$ ). K, Opeas minutum, spire apex (SMNS 101551, from Edelbeuren-Maurerkopf; $\mathrm{H}=1.8 \mathrm{~mm}$ ). L, Clausiliidae indet., spire apex (SMNS 101550, from Edelbeuren-Maurerkopf; $\mathrm{H}$ $=0.7 \mathrm{~mm}$ ). M. Clausiliidae indet., fragment of body whorl, apertural region (SMNS 101550, from Edelbeuren-Maurerkopf; fragment width = 2.1 mm). N, Triptychia sp., spire apex (SMNS 101548, from Edelbeuren-Maurerkopf; H= 2.6 mm). O-P, Archaeozonites costatus (SMNS 101559, from Edelbeuren-Schlachtberg; H = $17.3 \mathrm{~mm}, \mathrm{D}=27.0 \mathrm{~mm}$ ). Q, Klikia sp. (SMNS 101552, from Edelbeuren-Maurerkopf; D = $11.2 \mathrm{~mm}$ ). R-T, Palaeotachea renevieri (SMNS 101562, from Heselsberg; $\mathrm{H}=11.2 \mathrm{~mm}, \mathrm{D}=17.1 \mathrm{~mm}$ ). U-W, Megalotachea silvana, unusually large specimen (SMNS 101609, from Bonlanden; $\mathrm{H}=19.0 \mathrm{~mm}, \mathrm{D}=28.0 \mathrm{~mm}$ ). 
taxonomical value and, therefore, identification of the present specimens cannot proceed further than genus level. As pointed by Harzhauser et al. (2014), fossil Deroceras are known since the Miocene, but are often identified as either Limax Linnaeus, 1758 or Milax Gray, 1855.

Paleoecological remarks. Deroceras is known from a broad range of habitats, from the more usual open grasslands to forests, including wetlands and anthropically disturbed areas (Welter-Schultes, 2012; Rowson et al., 2014).

\author{
Class BIVALVIA Linnaeus, 1758 \\ Order UNIONOIDA Stoliczka, 1871 \\ Family MARGARITIFERIDAE Henderson, 1929 (1910)
}

Margaritifera Schumacher, 1816

Margaritifera flabellata (Goldfuss, 1837)

(Figures 3E-G)

Synonymy. See Schneider \& Prieto (2011) and complement: 1970 Plicatibaphia flabellata: Starobogatov, p. 61.

1999 Unio sp. vel Margaritifera sp.: Sach, p. 16; Sach et al., 2003, p. 6; Sach, 2014, p. 27.

2014 Unio sp. vel Margaritifera sp.: (in part) Sach, p. 81. 1999 Margaritifera flabellata: Sach, p. 20; Sach, 2014, p. 30. 2011 Margaritifera (Pseudunio) flabellata: Schneider \& Prieto, p. 4, figs. 3-6.

2012 Plicatibaphia bavarica: Grim et al., p. 19, pl. 1.

Material examined. SMNS 101629 (three specimens), 101630 (five specimens), 101631 (two specimens), 101632 (three specimens), 101633 (seven specimens), 101634 (two specimens), 101635 (three specimens), 101636 (two specimens), 101637 (three specimens), 101638 (one specimen), 101639 (one specimen), 101640 (two specimens), 101656 ( 10 specimens).

Occurrence. Wannenwaldtobel 2, Tobel OelhaldeNord, Tobel Oelhalde-Süd, Edelbeuren-Schlachtberg, Binnrot, Bonlanden, Edelbeuren-Maurerkopf, Heselsberg, Burgerbachtobel 1, Schmalegger Tobel, Lattentobel and Altenstadt-Untereichen.

Description. Valve elliptical to reniform; middle third of valve length slightly compressed in height; 35\% longer than taller. Shell inflated, width $\sim 40 \%$ of total shell length. External surface frequently smooth, sometimes covered by traces of growth lines and deep and worn plicae at posterior margin of valves. Internal surface smooth, without scars preserved. Umbones wide and low, $\sim 20 \%$ of total valve length and $~ 5 \%$ of total valve height, located between anterior and middle third of shell length. Ligament external, long and narrow, length $~ 60 \%$ of total shell length. Right hinge composed by two pseudocardinal teeth, disposed anteriorly to umbones axis, on a dental shelf; dental shelf triangular, located between anterior and dorsal shell margins; shape approximately fusiform; length $\sim 60 \%$ of total valve height; anterior tooth long and narrow, length $\sim 30 \%$ of dental shelf length, laminar, distanced from posterior tooth $\sim 1.5$ times its own width; posterior tooth short and rounded, $\sim 1 / 2$ anterior tooth total length. Left hinge with two pseudocardinal teeth, disposed anteriorly to umbones axis, on a dental shelf; dental shelf triangular, wide, length $\sim 90 \%$ of total valve height; anterior tooth long, length same as dental shelf width, pyramidal, dorsal to middle portion wide and strong, middle and anterior portion laminar, away from posterior tooth $c a$. twice its own width; posterior tooth strong and wide, triangular and flat, length same as anterior tooth, greatest width $\sim 10 \%$ total dental shelf length.

Discussion. Margaritifera flabellata is characteristic of the late Miocene in a wide range of Central European localities and shows a constancy of overall shell forms (Kókay, 2006; Schneider \& Prieto, 2011). Moreover, this species shows some variety in shell ornamentation, which is deemed to be due to preservation issues rather than related to distinct environmental conditions (Schneider \& Prieto, 2011).

Paleoecological remarks. Recent European Margaritifera species inhabit fast flowing and well oxygenated waters (Harzhauser \& Tempfer, 2004), with the larger M. auricularia (Spengler, 1793) usually occurring in large rivers (WelterSchultes, 2012). The fossil M. flabellata more closely resembles the recent $M$. margaritifera (Linnaeus, 1758), which usually is found in clear running waters (Welter-Schultes, 2012). Apparently, the conditions in the present sites during the Miocene were very favorable to M. flabellata: the species is widespread, occurring in nearly all the mollusk-bearing localities presented here (Table 1), reasonably abundant and reaches large sizes; moreover, there is an equal proportion of articulated and disarticulated specimens, indicating that the species is authochtonous. Given the environmental interpretation of the sites (Sach, 1999; see also discussion section below), $M$. flabellata seems to have had different preferences than its living relatives. Moreover, this species is also known from some paludal Miocene environments (Schneider \& Prieto, 2011).

Family UNIONIDAE Fleming, 1828

Unio Philipsson, 1788

Unio kirchbergensis Krauss, 1852

(Figures 3J-K)

1852 Unio kirchbergensis Krauss, p. 152, figs. 5a-c; Pictet, 1855, p. 529; Lea, 1870, p. 65.

2014 Unio sp. vel Margaritifera sp.: (in part) Sach, p. 81.

Material examined. SMNS 101650 (one specimen).

Occurrence. Altenstadt-Untereichen.

Description. Valve elliptical, $\sim 40 \%$ longer than taller. Valve width $\sim 20 \%$ of total valve length. External surface mostly smooth, with traces of growth lines on middle and ventral portions. Internal surface smooth, without scars preserved. Umbones wide and low, 25\% of total valve length and 5\% of total valve height, located between anterior and middle third of length. Left hinge with two cardinal teeth disposed parallel 


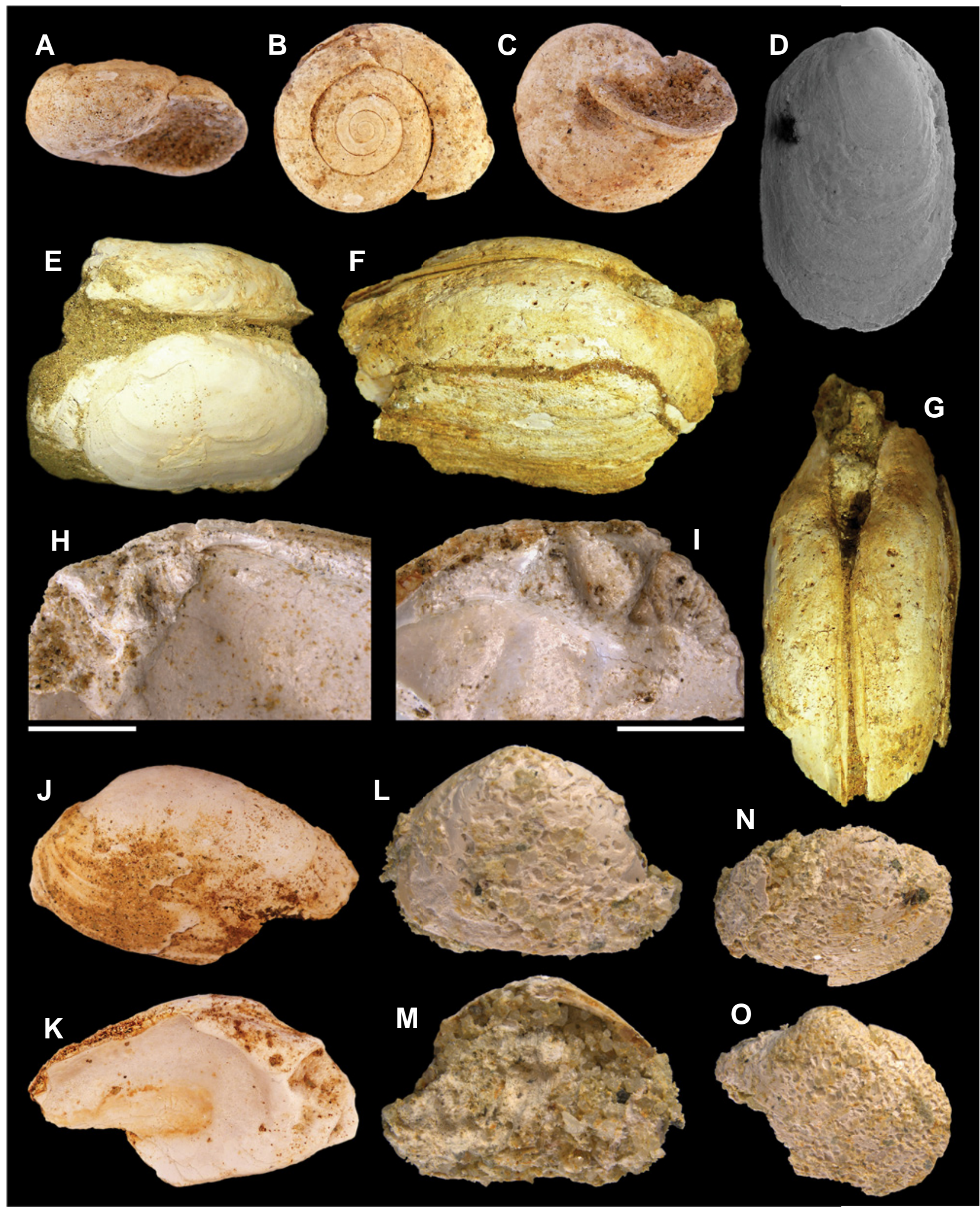

Figure 3. A-C, Pseudochloritis incrassata (SMNS 101585, from Edelbeuren-Schlachtberg; H = $15.3 \mathrm{~mm}, \mathrm{D}=26.0 \mathrm{~mm}$ ). D, Deroceras sp. (SMNS 101554, from Edelbeuren-Maurerkopf; H=9.5 mm, D =6.1 mm). E, Margaritifera flabellata, left valve (SMNS 101656, from AltenstadtUntereichen; $\mathrm{L}=66.0 \mathrm{~mm}$ ). F-G, M. flabellata, respectively, lateral (right valve) and dorsal view of closed specimen (SMNS 101632, from Edelbeuren-Schlachtberg; L = 65.1 mm). H, M. flabellata, detail of right hinge (SMNS 101633, specimen \#1, from Edelbeuren-Schlachtberg; scale bar = $1 \mathrm{~cm}$ ). I, M. flabellata, detail of left hinge (SMNS 101633, specimen \#2, from Edelbeuren-Schlachtberg; scale bar = $1 \mathrm{~cm}$ ). J-K, Unio kirchbergensis, respectively, external and internal view of left valve (SMNS 101650, from Altenstadt-Untereichen; $\mathrm{L}=48.8 \mathrm{~mm}$ ). L-M, Sphaerium aff. S. rivicola, respectively, external and internal view of left valve (SMNS 101628, specimen \#1, from Binnrot; L = $7.7 \mathrm{~mm}$ ). N, Sphaerium aff. S. rivicola, right valve (SMNS 101628, specimen \#2, from Binnrot; $L=10.7 \mathrm{~mm}$ ). O, Sphaerium aff. S. rivicola, right valve (SMNS 101628 , specimen \#3, from Binnrot; $L=10.3 \mathrm{~mm}$ ). 
to umbones axis, on a dental shelf. Dental shelf long and straight, length $\sim 90 \%$ of total valve height, located between dorsal and anterior margins; shape follows that of valve's margins. Anterior tooth triangular, flat, $~ 35 \%$ shorter than dental shelf width, posterior end wider than anterior; touches posterior end of posterior tooth; greatest width 30\% of total dental shelf length; distant from posterior by roughly its own width. Posterior tooth long, wide; length equal to dental shelf width; greatest width $~ 55 \%$ of total dental shelf length.

Discussion. This single specimen is externally identical to a much worn Margaritifera flabellata, but the hinge is wellpreserved and its proportions are very peculiar. The anterior tooth is very flat and triangular, while in M. flabellata it is pyramidal with disproportional sides. The posterior tooth is broad, occupying almost the entire posterior portion of the dental shelf area, while $M$. flabellata presents a posterior tooth with roughly half this size. The present specimen compares fittingly with the syntypes of Unio kirchbergensis (SMNS 25504/2005), a species known only from its type locality, Kirchberg an der Iller (southern Germany), from the late early Miocene Kirchberg Formation.

Paleoecological remarks. Recent Unio species have a wide range of habitats; nevertheless, they are more usually found in rivers, avoiding small water bodies, and do not tolerate desiccation (Welter-Schultes, 2012).

Order VENEROIDA H. Adams \& A. Adams, 1856
Family SPHAERIIDAE Deshayes, 1855 (1820)

Sphaerium Scopoli, 1777

Sphaerium aff. S. rivicola (Lamarck, 1818)

(Figures 3L-O)

Synonymy. See Schneider \& Prieto (2011) and complement: 1999 Sphaerium sp.: Sach, p. 22; Sach, 2014, p. 31.

2011 Sphaerium (Amesoda) rivicola: Schneider \& Prieto, p. 9, figs. 7A-C.

Material examined. SMNS 101628 (four specimens). Occurrence. Binnrot.

Description. Valve triangular to elliptical, 30\% longer than taller. Valves flattened, width $\sim 10 \%$ of total shell length. External surface with growth lines. Internal surface not observable due to poor preservation. Umbones wide and low, prominent from shell outline, located on the middle of anterior portion of valve; 25\% valve total length and $\sim 10 \%$ total valve height. Ligament external, long and narrow; $50 \%$ total valve length. Hinge poorly preserved; cardinal tooth not observable; lateral tooth on middle portion of both sides of dorsal margin.

Discussion. Lueger (1979) reported the first Late Miocene record of this species from Vienna Basin, Austria, justifying his identification as Sphaerium rivicola, a recent species, due to the species' occurrence as Pleistocene fossils. Later, Schneider \& Prieto (2011) reported the species from the Late Miocene of southern Germany. The present specimens are very similar in overall shape and proportions to those reported by Lueger (1979) and Schneider \& Prieto (2011), being much more reminiscent of those reported by the latter. The present specimens are anteroventrally elongated, with less pronounced umbones, while Lueger's are nearly round, with a posterior carina and pronounced umbones. Conchological features apart, geographical and stratigraphical distributions are still problematic: S. rivicola is a recent European species. It is well know that recent freshwater bivalves can have broad geographical distributions, throughout rivers and others small water bodies (e.g. Pereira et al., 2014), but ca. $15 \mathrm{Ma}$ separate the recent $S$. rivicola from the fossils. Therefore, here we prefer a more conservative classification of the present material, linking the present material to the specimens of Schneider \& Prieto (2011), due to their shared similarity in conchological features and stratigraphical and geographical proximity. A thorough revision of all the available fossil material is necessary to a better definition of Sphaerium morphotypes occurring in the European Miocene.

Paleoecological remarks. The valves are poorly preserved and disarticulate and the numbers of specimens is too small for any taphonomical inference. Recent Sphaerium species show a remarkable tolerance to wide ranges of dissolved oxygen in water, from rapid and well-oxygenated waters to temporary and hypoxic water bodies (Cummings \& Graf, 2010; Welter-Schultes, 2012).

\section{DISCUSSION}

The poor preservation of part of the material studied here is problematic for taxonomy; therefore, for fragmentary and/ or deformed specimens and internal molds identification could not proceed further than genus level (or family level for the clausiliid). Table 1 lists all the fossil sites in the districts of Biberach, Ravensburg and Neu-Ulm and the species found in each one. Table 1 also lists the abundance of each species for each site, but it should be noted that the collection of the molluscan specimens was not controlled for this and techniques for recovering microgastropods (usually abundant in other Miocene outcrops in southern Germany) were not used.

The species list presented here (Table 1) is consistent with those presented by Sach $(1999,2014)$. Nevertheless, some new occurrences were also noted here: Gyraulus applanatus, Opeas minutum, Archaeozonites costatus, Palaeotachea renevieri, Triptychia sp. and Unio kirchbergensis. Moreover, a few misidentifications listed by Sach $(1999,2014)$ and Sach et $a l$. (2003) were rectified here: the single specimen previously identified as Pomatias conicus is a spire apex of Lymnaea dilatata and, likewise, the single specimen of Valvata sp. is a misidentification of a fragment of Archaeozonites costatus.

Sach (1999) presented a paleoenvironmental interpretation of the fossiliferous horizons of the Biberach district, proposing a large meandering river system surrounded by alluvial and gallery forests. This interpretation was based upon the crossstratification lithology of the finely grained sandy sediments and on the evaluation of the fossil remains found, especially 
Table 1. Molluscan species occurring in the middle Miocene fossil sites of the districts of Biberach, Ravensburg and Neu-Ulm. Fossil sites (names according to Sach, 1999, 2014): A Wannenwaldtobel 1 and 2; B, Tobel Oelhalde-Nord; C, Tobel Oelhalde-Süd; D, Edelbeuren-Schlachtberg; E, Binnrot; F, Bonlanden; G, Edelbeuren-Maurerkopf; H, Heselsberg; I, Auttagershofen; J, Burgerbachtobel 1; K, Schmalegger Tobel; L, Lattentobel; $\mathbf{M}$, Altenstadt-Untereichen. Number of specimens found: $\mathbf{x}, 1-10$ specimens; $\mathbf{x x}, 11-60$ specimens; $\mathbf{x x x},>60$ specimens. An " $\mathbf{S}$ " indicates a species listed by Sach $(1999,2014)$ that could not be found in the SMNS collection (specimens were too fragmentary and were not collected or kept).

\begin{tabular}{|c|c|c|c|c|c|c|c|c|c|c|c|c|c|}
\hline \multirow[b]{2}{*}{ Species } & \multicolumn{9}{|c|}{ BIBERACH } & \multicolumn{3}{|c|}{ RAVENSBURG } & \multirow{2}{*}{$\frac{\text { NEU-ULM }}{\mathrm{M}}$} \\
\hline & $\mathrm{A}$ & B & $\mathrm{C}$ & $\mathrm{D}$ & $\mathrm{E}$ & $\mathrm{F}$ & G & $\mathrm{H}$ & I & $\mathrm{J}$ & $\mathrm{K}$ & $\mathrm{L}$ & \\
\hline \multicolumn{14}{|l|}{ CAENOGASTROPODA } \\
\hline Bithynia sp. & & & & & & & $\mathrm{xxx}$ & & $\mathrm{S}$ & & & & \\
\hline Pomatias sp. & & & & & & & & & & $\mathrm{x}$ & & & \\
\hline Tinnyea laureae & & & & & & & & & & & & & $\mathrm{x}$ \\
\hline \multicolumn{14}{|l|}{ NERITIMORPHA } \\
\hline Theodoxus sp. & & & & & & & & & & & & & $\mathrm{x}$ \\
\hline \multicolumn{14}{|l|}{ HYGROPHILA } \\
\hline Galba cf. G. dupuyiana & $\mathrm{xxx}$ & & & & & & $\mathrm{x}$ & & $\mathrm{x}$ & & & & \\
\hline Gyraulus applanatus & $\mathrm{xxx}$ & & & & & & $\mathrm{x}$ & & & & & & \\
\hline Lymnaea dilatata & $\mathrm{x}$ & & $\mathrm{x}$ & & & $\mathrm{x}$ & $\mathrm{xxx}$ & $\mathrm{x}$ & & $\mathrm{x}$ & & & $\mathrm{x}$ \\
\hline Planorbarius cornu & $\mathrm{x}$ & $\mathrm{x}$ & $\mathrm{x}$ & $\mathrm{x}$ & & $\mathrm{x}$ & $\mathrm{x}$ & $\mathrm{x}$ & $\mathrm{x}$ & & & & $\mathrm{x}$ \\
\hline \multicolumn{14}{|l|}{ STYLOMMATOPHORA } \\
\hline Archaeozonites costatus & & & & $\mathrm{x}$ & $\mathrm{x}$ & & $\mathrm{x}$ & & & & & & $\mathrm{x}$ \\
\hline Clausiliidae indet. & & & & & & & $\mathrm{x}$ & & & & & & \\
\hline Deroceras sp. & $\mathrm{x}$ & & & $\mathrm{S}$ & & $\mathrm{xx}$ & $\mathrm{xxx}$ & & & $\mathrm{xx}$ & & & \\
\hline Klikia sp. & & & & & & $\mathrm{S}$ & $\mathrm{xxx}$ & $\mathrm{x}$ & & $\mathrm{S}$ & & & $\mathrm{x}$ \\
\hline Megalotachea silvana & $\mathrm{xx}$ & $\mathrm{xx}$ & $\mathrm{xx}$ & $\mathrm{xx}$ & $\mathrm{x}$ & $\mathrm{xx}$ & $\mathrm{xxx}$ & $\mathrm{x}$ & $\mathrm{x}$ & $\mathrm{x}$ & & & $\mathrm{xx}$ \\
\hline Opeas minutum & & & & & & & $\mathrm{x}$ & & & & & & \\
\hline Palaeotachea renevieri & & $\mathrm{xx}$ & $\mathrm{xx}$ & & & $\mathrm{xx}$ & $\mathrm{xx}$ & $\mathrm{x}$ & $\mathrm{x}$ & $\mathrm{x}$ & & & \\
\hline Pseudochloritis incrassata & $\mathrm{x}$ & $\mathrm{x}$ & $\mathrm{x}$ & $\mathrm{xx}$ & $\mathrm{x}$ & & $\mathrm{xxx}$ & $\mathrm{x}$ & & $\mathrm{S}$ & $\mathrm{x}$ & & $\mathrm{x}$ \\
\hline Triptychia sp. & & & & & & & $\mathrm{x}$ & & & & & & \\
\hline \multicolumn{14}{|l|}{ BIVALVIA } \\
\hline Margaritifera flabellata & $\mathrm{x}$ & $\mathrm{x}$ & $\mathrm{x}$ & $\mathrm{x}$ & $\mathrm{x}$ & $\mathrm{x}$ & $\mathrm{x}$ & $\mathrm{x}$ & & $\mathrm{x}$ & $\mathrm{x}$ & $\mathrm{x}$ & $\mathrm{x}$ \\
\hline Sphaerium aff. S. rivicola & & & & & $\mathrm{x}$ & & & & & & & & \\
\hline Unio kirchbergensis & & & & & & & & & & & & & $\mathrm{x}$ \\
\hline
\end{tabular}

mammals, fishes, turtles and mollusks. Shells of freshwater mollusks would have been concentrated by the streams' waters, while periodical floods would have transported the shells of terrestrial snails from the neighboring area into the river system. The calcareous sediments (marls and freshwater limestones), however, were calmly deposited in the stagnant waters of ponds and small lakes. A very similar fluvial environment of meandering rivers, still water habitats and neighboring alluvial forests can also be assumed for the fossiliferous horizons in the districts of Ravensburg and Neu-Ulm.

The molluscan freshwater fauna, as already observed by Sach (1999), is consistent with rivers, oxbow lakes and ponds. Bithynia and the freshwater pulmonates indicate still or slowflowing waters, including perhaps short-lived shallow water bodies. This is in accordance with the slow-flowing streams and ponds proposed by Sach (1999), with Bithynia and the freshwater pulmonates inhabiting the well vegetated areas close to the margins.

In the immediate vicinity of the water bodies, there were humid alluvial and gallery forests that went through periodical floods (Sach, 1999). These forests would have provided favorable conditions for the land snails (Opeas, the clausilioids and supposedly Archaeozonites) that prefer warmer and more humid environments. There is also indication, mainly based on plant and mammal remains, of drier forests and open areas (Sach, 1999), but, despite snails of such habitats being rather common in other German Miocene sites (e.g. Randeck Maar; Salvador et al., 2015), there is no such fauna in the present material. The two fossil genera Pseudochloritis and Klikia supposedly inhabited more exposed areas (Lueger, 1981; Binder, 2008; Moser et al., 2009) and they are very abundant in one of the sites studied here, namely Edelbeuren-Maurerkopf. These areas would be located farther away from the water bodies (and perhaps on raised ridges; Sach, 1999).

\section{ACKNOWLEDGEMENTS}

We are deeply grateful to K. Wolf-Schwenninger (SMNS) for the SEM images; to M. Battenstein (SMNS) for the preparation of the Sphaerium specimens; to M.W. Rasser and R. Ziegler (SMNS) for the help in tracking down the material; to O. Höltke (SMNS) for the help with the "Cepaea" specimens; to M. Harzhauser (NHMW) and S. Schneider (University of Cambridge) for the help with the Polish bibliography and comments on the taxonomy of Margaritifera flabellata; and to B. Reichenbacher (BSPG), G. Manganelli (Università di Siena), L. Satour (University of Oran) and an anonymous reviewer for their helpful comments on the manuscript. This work was partly supported by a doctorate grant from Conselho Nacional de Desenvolvimento Científico e Tecnológico, Brazil (CNPq) to R.B.S. (process \#245575/2012-0). 


\section{REFERENCES}

Bandel, K. 2001. The history of Theodoxus and Neritina connected with description and systematic evaluation of related Neritimorpha (Gastropoda). Mitteilungen aus dem GeologischPaläontologischen Institut der Universität Hamburg, 85:65-164.

Berz, K.C. \& Jooss, C.H. 1927. Über die Altersstellung der tertiären Schichten (Süßwasserablagerungen und bunter Breccia) von Oggenhausen bei Heidenheim a. d. Brenz. Centralblatt für Mineralogie, Geologie, Paläontologie, Abhandlungen B, 1927:193-208.

Binder, H. 2002. Die Land- und Süßwassergastropoden aus dem Karpatium des Korneuburger Beckens (Niederösterreich; Untermiozän). Beiträge zur Paläontologie, 27:161-203.

Binder, H. 2004. Terrestrial, freshwater and brachyhaline Gastropoda from the Lower Miocene deposits of Oberdorf (Styria, Austria). Annalen des Naturhistorischen Museums in Wien, 105A:189-229.

Binder, H. 2008. The systematic positions of the genera Pseudochloritis C. Boettger 1909 and Joossia Pfeffer 1929. Archiv für Molluskenkunde, 137:167-193. doi:10.1127/arch. moll/0003-9284/137/167-193

Böttcher, R.; Heizmann, E.P.J.; Rasser, M.W. \& Ziegler, R. 2009. Biostratigraphy and palaeoecology of a Middle Miocene (Karpathian, MN 5) fauna from the northern margin of the North Alpine Foreland Basin (Oggenhausen 2, SW' Germany). Neues Jahrbuch für Geologie und Paläontologie, Abhandlungen, 254:237-260.

Bouchet, P.; Rocroi, J.-P.; Frýda, J.; Hausdorf, B.; Ponder, W.; Valdés, Á. \& Warén, A. 2005. Classification and nomenclator of gastropod families. Malacologia, 47:1-397.

Braun, A. 1851. Darstellung der geognostischen Verhältnisse des Mainzer Beckens und seiner fossilen Fauna und Flora. In: F.A. Walchner (ed.) Handbuch der Geognosie, Groos, p. 1112-1169.

Brongniart, M. 1810. Sur des terrains qui paraissent avoir été formés sous l'eau douce. Annales du Muséum d'Histoire Naturelle, 15:357-405.

Bunje, P.M.E. 2005. Pan-European phylogeography of the aquatic snail Theodoxus fluviatilis (Gastropoda: Neritidae). Molecular Ecology, 14:4323-4340. doi:10.1111/j.1365-294x.2005.02703.x

Chapuis, E.; Trouve, S.; Facon, B.; Degen, L. \& Goudet, J. 2007. High quantitative and no molecular differentiation of a freshwater snail (Galba truncatula) between temporary and permanent water habitats. Molecular Ecology, 16:3484-3496. doi:10.1111/j.1365-294x.2007.03386.x

Cummings, K.S. \& Graf, D.L. 2010. Mollusca: Bivalvia. In: J.H. Thorp \& A.P. Covich (eds.) Ecology and classification of North American freshwater invertebrates, Elesevier, p. 309-384. doi:10.1016/B978-0-12-374855-3.00011-X

Ehrat, H. \& Jooss, C.H. 1921. Das Alter der vulkanischen Tuffe im Kirchheim-Uracher Gebiet und im Hegau. Geologische und Paläontologische Mitteilungen, 1:1-8.

Fischer, J.-C. 2000. La malacofaune de Sansan. Mémoires du Muséum National d'Histoire Naturelle, 183:129-154.

Gall, H. 1980. Eine Gastropodenfauna aus dem Landshuter Schotter der Oberen Süßwassermolasse (Westliche Paratethys, Badenien) von Gündlkofen/Niederbayern. Mitteilungen der Bayerischen Staatssammlung für Paläontologie und historische Geologie, 20:51-77.

Glöer, P. 2002. Süsswassergastropoden Nord-und Mitteleuropas: Bestimmungsschlüssel, Lebensweise, Verbreitung. Hackenheim, ConchBooks, 327 p.
Goldfuss, G.A. 1826-1844. Petrefacta Germaniae, tam ea quae in Museo Universitatis Regiae Borussiae Friedericiae Wilhelmae Rhenanae servantur quam alia quaecumque in Museis Hoeninghusiano, Münsterniano, aliisque extant, iconibus et descriptionibus illustrata, 2. Düsseldorf, Arnz, 312 p.

Górka, M. 2008. Shell colour pattern in two fossil helicid snails, Tropidomphalus incrassatus (Klein, 1853) and Cepaea sylvestrina gottschicki Wenz, 1919, from the Middle Miocene of Poland. Acta Geologica Polonica, 58:105-111.

Gottschick, F. \& Wenz, W. 1916. Die Sylvanaschichten von Hohenmemmingen und ihre Fauna. Nachrichtsblatt der Deutschen Malakozoologischen Gesellschaft, 48:17-31, 55-74, 97-113.

Grim, M.C.; Renker, C.; Forman, M. \& Engel, T. 2012. Erste bestimmbare Mollusken aus der obermiozänen EppelsheimFormation (Tertiär, Tortonium, Rheinhessen): Plicatibaphia bavarica (Modell, 1938) (Bivalvia: Unionida,Margaritiferidae). Mainzer naturwissenschafliches Archiv, 49:13-24.

Harzhauser, M.; Kowalke, T. \& Mandic, O. 2002. Late Miocene (Pannonian) gastropods of Lake Pannon with special emphasis on early ontogenetic development. Annalen des Naturhistorischen Museums in Wien, 103A:75-141.

Harzhauser, M.; Neubauer, T.A.; Gross, M. \& Binder, H. 2014. The early Middle Miocene mollusc fauna of Lake Rein (Eastern Alps, Austria). Palaeontographica Abteilung A, 302:1-71.

Harzhauser, M.; Neubauer, T.A.; Mandic, O.; Zuschin, M. \& Ćorić, S. 2012. A Middle Miocene endemic freshwater mollusc assemblage from an intramontane Alpine lake (Aflenz Basin, Eastern Alps, Austria). Paläontologische Zeitschrift, 86:23-41. doi:10.1007/s12542-011-0117-x

Harzhauser, M. \& Tempfer, P.M. 2004. Late Pannonian wetland ecology of the Vienna Basin based on Molluscs and lower vertebrate assemblage (Late Miocene, MN 9, Austria). Courier Forschungs-Institut Senckenberg, 246:55-68.

Höltke, O. \& Rasser, M.W. 2015. Pseudochloritis insignis - a peculiar large land-snail from the Miocene of SW Germany: taxonomic status and census of morphologically related forms. Journal of Conchology, 42:1-12.

Kadolsky, D. 1995. Stratigraphie und Molluskenfaunen von „Landschneckenkalk“ und „Cerithienschichten“ im Mainzer Becken (Oberoligozän bis Untermiozän?), 2: Revision der aquatischen Mollusken des Landschneckenkalkes. Archiv für Molluskenkunde, 124:1-55.

Klein, R. 1846. Conchylien der Süßwasserkalkformation Wüttembergs. Jahreshefte des Vereins für Vaterländische Naturkunde in Württemberg, 2:60-116.

Klein, R. 1853. Conchylien der Süsswasserkalkformationen Württembergs. Jahreshefte des Vereins für vaterländische Naturkunde in Württemberg, 9:203-223.

Kókay, J. 2006. Nonmarine mollusc fauna from the Lower and Middle Miocene, Bakony Mts, W Hungary. Geologica Hungarica, Series Palaeontologica, 56:3-196.

Kowalke, T. \& Reichenbacher, B. 2005. Early Miocene (Ottnangian) Mollusca of the Western Paratethys - ontogenetic strategies and palaeo-environments. Geobios, 38:609-635. doi:10.1016/j. geobios.2004.03.006

Krauss, F. 1852. Die Mollusken der Tertiär-Formation von Kirchberg an der Iller. Jahreshefte des Vereins für vaterländische Naturkunde in Württemberg, 8:136-157.

Lamarck, J.B. 1818. Histoire naturelle des animaus sans vertèbres, présentant les caractères généraux et particuliers de ces animaux, leus distribution, leurs classes, leurs families, leurs genres, et la citation des principales espèces qui s'y rapportent. Tome cinquième. Paris, Deterville \& Verdiere, 612 p. 
Lea, I. 1870. A synopsis of the family Unionidae. $4^{\text {th }}$ ed. Philadelphia, Henry C. Lea, 184 p.

Lueger, J.P. 1979. Rezente Flußmollusken im Pannon (O. Miozän) des Wiener Beckens (Österreich). Sitzungsberichte, Österreichische Akademie des Wissenschaften, Mathematischenaturwissenschaftliche Klasse, Abteilung 1, 188:87-95.

Lueger, J.P. 1981. Die Landschnecken im Pannon und Pont des Wiener Beckens, I. Systematik. II. Fundorte, Stratigraphie, Faunenprovinzen. Denkschriften der Akademie der Wissenschaften, mathematisch-naturwissenschaftliche Klasse, 120:1-124.

Maillard, G. 1892. Monographie des mollusques tertiaires terrestres er fluviatiles de la Suisse. Abhandlung der Schweizer Paläontologische Gesellschaft, 18:1-127.

Mathéron, P. 1842-1843. Catalogue méthodique et descriptif des corps organisés fossiles du Département des Bouches-du-Rhône et lieux circonvoisins; précédé d‘un mémoire sur les terrains supérieurs au Grès Bigarré du S.E. de la France. Répertoire des Travaux de la Société de Statistique de Marseille, 6:1-269.

Mikuz, V. \& Pavsic, J. 2000. Brotia (Tinnyea) escheri (Brongniart) iz miocenskih plasti pri Tunjicah. Geologija, 43:43-53.

Moser, M.; Niederhöfer, H.-J. \& Falkner, G. 2009. Continental molluscs of the fossil site Sandelzhausen (Miocene; Upper Freshwater Molasse from Bavaria) and their value for palaeoecological assessment. Paläontologische Zeitschrift, 83:25-54. doi:10.1007/s12542-009-0013-9

Neubauer, T.A.; Kroh, A.; Harzhauser, M.; Georgopoulou, E. \& Mandic, O. 2014. Synopsis of valid species-group taxa for freshwater Gastropoda recorded from the European Neogene. ZooKeys, 435:1-6. doi:10.3897/zookeys.435.8193

Nordsieck, H. 2007. Worldwide Door Snails (Clausiliidae), Recent and Fossil. Hackenheim, ConchBooks, 214 p.

Nordsieck, H. 2014. Annotated check-list of the genera of fossil land snails (Gastropoda: Stylommatophora) of western and central Europe (Cretaceous-Pliocene), with description of new taxa. Archiv für Molluskenkunde, 143:153-185. doi:10.1127/arch. moll/1869-0963/143/153-185

Noulet, J.B. 1854. Mémoire sur les coquilles fossiles des terrains d'eau douce du sud-ouest de la France. Paris, Victor Masson, $127 \mathrm{p}$.

Papp, A. 1953. Die Molluskenfauna des Pannon im Wiener Becken. Mitteilungen der geologischen Gesellschaft in Wien, 44:85-222.

Pereira, D.; Mansur, M.C.D.; Duarte, L.D.S.; Oliveira, A.S.; Pimpão, D.M.; Callil, C.T.; Ituarte, C.; Parada, E.; Peredo, S.; Darrigran, G.; Scarabino, F.; Clavijo, C.; Lara, G.; Miyahira, I.C.; Rodriguez, M.T.R. \& Lasso, C. 2014. Bivalve distribution in hydrographic regions in South America: historical overview and conservation. Hydrobiologia, 735:15-44. doi:10.1007/ s10750-013-1639- $x$

Pictet, F.J. 1855. Traité de Paléontologie ou Histoire Naturelle des Animaux Fossiles Considérés dans leurs rapports Zoologiques et Géologiques. $2^{\text {nd }}$ ed. Paris, J.-B. Baillière, 654 p.

Rasser, M.W.; Bechly, G.; Böttcher, R.; Ebner, M.; Heizmann, E.P.J.; Höltke, O.; Joachim, C.; Kern, A.K.; Kovar-Eder, A.J.; Nebelsick, J.H.; Roth-Nebelsick, A.; Schoch, R.R.; Schweigert, G. \& Ziegler, R. 2013. The Randeck Maar: palaeoenvironment and habitat differentiation of a Miocene lacustrine system. Palaeogeography, Palaeoclimatology, Palaeoecology, 392:426453. doi:10.1016/j.palaeo.2013.09.025

Reichenbacher, B. 1989. Feinstratigraphische Gliederung der Kirchberger Schichten (Unter-Miozän) an der Typuslokalität Illerkirchberg bei Ulm. Geologica Bavarica, 94:135-177.
Rowson, B.; Turner, J.; Anderson, R. \& Symondson, B. 2014. Slugs of Britain and Ireland: identific ation, understanding and control. Telford, FSC, $136 \mathrm{p}$.

Sach, V.J. 1999. Litho- und biostratigraphische Untersuchungen in der Oberen Süßwassermolasse des Landkreises Biberach an der Riß (Oberschwaben). Stuttgarter Beiträge zur Naturkunde, Serie B, 276:1-167.

Sach, V.J. 2014. Fossilienkatalog der Oberen Süßwassermolasse (OSM), Brackwassermolasse (BM), Oberen Meeresmolasse (OMM) und der Unteren Süßwassermolasse (USM) in Südwestdeutschland: Landkreis Biberach an der Riß, Landkreis Ravensburg und Bodenseekreis, Ulmer/Neu-Ulmer Gegend, Landkreis Sigmaringen. Documenta Naturae, 70:1-112.

Sach, V.J.; Gaudant, J.; Reichenbacher, B. \& Böhme, M. 2003. Die Fischfaunen der Fundstellen Edelbeuren-Maurerkopf und Wannenwaldtobel 2 (Miozän, Obere Süßwassermolasse, SWDeutschland). Stuttgarter Beiträge zur Naturkunde, Serie B, 334:1-25.

Salvador, R.B. 2013. The fossil pulmonate snails of Sandelzhausen (Early/Middle Miocene, Germany): Succineidae, Testacelloidea and Helicoidea. Zootaxa, 3721:157-171. doi:10.11646/ zootaxa.3721.2.3

Salvador, R.B. 2014. The fossil land and freshwater snails of Gündlkofen (Middle Miocene, Germany). Zootaxa, 3785:271287. doi:10.11646/zootaxa.3785.2.9

Salvador, R.B. \& Rasser, M.W. 2014. The fossil pulmonate snails of Sandelzhausen (Early/Middle Miocene, Germany): Hygrophila, Punctoidea and limacoids. Archiv für Molluskenkunde, 143:187202. doi:10.1127/arch.moll/1869-0963/143/187-202

Salvador, R.B.; Rasser, M.W. \& Höltke, O. (2015). Fossil gastropods from Miocene Lake Randeck Maar and its hinterland (SW Germany). Neues Jahrbuch für Geologie und Paläontologie, Abhandlungen, 277:251-273.

Sandberger, F. 1870-1875. Die Land- und Süßwasser-Conchylien der Vorwelt. Wiesbaden, C.W. Kreidel, 1000 p.

Schlickum, W.R. 1964. Die Molluskenfauna der Süßbrackwassermolasse Niederbayerns. Archiv für Molluskenkunde, 93:1-68.

Schlickum, W.R. 1966. Die Molluskenfauna der Kirchberger Schichten des Jungholzes bei Leipheim/Donau. Archiv für Molluskenkunde, 95:321-335.

Schlickum, W.R. 1970a. Die Molluskenfauna der Kirchberger Schichten des Chiemsee-Gebietes. Geologica Bavarica, 63:143-158.

Schlickum, W.R. 1970b. Die Molluskenfauna der oberhelvetischen bis untertortonen brackischen und ausgesüßten Teile der Kohlenbohrungen zwischen Trostberg a. d. Alz und Tittmoning a. d. Salzach. Mitteilungen der Bayerischen Staatssammlung für Paläontologie und historische Geologie, 10:175-188.

Schlickum, W.R. 1976. Die in der pleistozänen Gemeindekiesgrube von Zwiefaltendorf an der Donau abgelagerte Molluskenfauna der Silvanaschichten. Archiv für Molluskenkunde, 107:1-31.

Schnabel, T. 2007. Die känozoischen Filholiidae Wenz 1923. Teil 4: Die eo- und oligozänen Vertreter der Gattung Triptychia, nebst Bemerkungen zur Ökologie und geo- bzw. stratigraphischen Verbreitung der Filholiidae sowie zur Evolution der Gattung Triptychia (Gastropoda, Pulmonata, Clausilioidea). Archiv für Molluskenkunde, 136:25-57. doi:10.1127/arch.moll/00039284/136/025-057

Schneider, S. \& Prieto, J. 2011. First record of an autochthonous community of fluviatile freshwater molluscs from the Middle/ Late Miocene Upper freshwater Molasse (southern Germany). Archiv für Molluskenkunde, 140:1-18. doi:10.1127/arch. moll/1869-0963/140/001-018 
Seemann, R. 1926. Geologische Untersuchungen in einigen Maaren der Albhochfläche. Jahreshefte des Vereins für vaterländische Naturkunde in Württemberg, 82:81-110.

Starobogatov, J.I. 1970. Fauna Molljuskov i zoogeografideskoe rajonirovanje kóntinental'nych vodoemov zemnogo sara. Leningrad, Akademija Nauk SSSR Zoologiceskij Institut, 372 p.

Steininger, F.; Ctyroky, P.; Hölzl, O.; Kókay, J.; Schlickum, W. R.; Schultz, O. \& Strauch, F. 1973. Die Mollusken des Ottnangien. In: A. Papp; F. Rögl \& J. Senes (eds.) Chronostratigraphie und Neostratotypen, Miozän der zentralen Paratethys, Ottnangien, p. 380-615.

Thomae, C. 1845. Fossile Conchylien aus den Tertiärschichten bei Hochheim und Wiesbaden gesammelt und im naturhistorischen Museum zu Wiesbaden aufgestellt. Jahrbuch des Nassauischen Vereins für Naturkunde, 2:125-166.

Welter-Schultes, F. 2012. European Non-marine Molluscs, a Guide for Species Identification. Göttingen, Planet Poster Editions, $679 \mathrm{p}$.
Wenz, W. 1923. Gastropoda extramarina tertiaria. In: C. Diener (ed.) Fossilium Catalogus I: Animalia, Junk, p. 1-1734.

Willig, M.R.; Presley, S.J.; Bloch, C.P. \& Alvarez, J. 2013. Population, community, and metacommunity dynamics of terrestrial gastropods in the Luquillo Mountains: a gradient perspective. Ecological Bulletins, 54:117-140.

Zettler, M.; Frankowski, J.; Bochert, R. \& Roehner, M. 2004. Morphological and ecological features of Theodoxus fluviatilis (Linnaeus, 1758) from Baltic brackish water and German freshwater populations. Journal of Conchology, 38:303-316.

Zilch, A. 1959-1960. Euthyneura. In: W. Wenz (ed.) Handbuch der Paläozoologie. Band 6, Teil, p. 1-835.

Zöbelein, H.K. 1954. Helvetische Landschnecken aus einem Knollenkalk bei Riedöschingen (Baden). Paläontologische Zeitschrift, 28:155-158.

Received in January, 2015; accepted in June, 2015. 